\title{
La investigación-creación en el contexto de la formación doctoral en diseño y creación en Colombia
}

\section{Research-creation in the context of doctoral training in design and creation in Colombia}

\author{
Sandra Johana Silva-Cañaveral ${ }^{1}$
}

Recibido: marzo 02 de 2016

Aceptado: mayo 10 de 2016

\section{Resumen}

Desde la experiencia derivada del propio camino investigativo y creativo adelantado durante la formación doctoral, en contraste con lo que implica realizar procesos de creación en el marco de mi práctica profesional como artista, propongo una reflexión sobre cómo investiga un/a creador/a en el campo de las artes visuales. Entre las transformaciones que están en juego hoy, está también la de volver a darle significado a una forma de conocimiento que esté entretejido a lo que somos, donde conocer signifique, primero que todo, aunque no exclusivamente, autoconocimiento, una transformación de sí, una experiencia, que de una $u$ otra manera pueda hacernos más "sabios" (más prudentes, en el sentido de la phronesis aristotélica). $Y$ en ese sentido, tal vez también pueda decirse que ser "doctor/a" no solo tiene que significar dominar los métodos de investigación propios de esa empresa anónima que es la ciencia, sino también, y sobre todo, lograr una experiencia que lo transforme a uno. Ser "doctor/a" debería también significar haber alcanzado cierto tipo de "virtud", haberse transformado en cierto tipo de persona, de mejor persona.

Palabras clave: investigación-creación, formación doctoral, artes visuales, diseño.

\begin{abstract}
I propose a reflection about how a creator does research in the field of visual arts, based on an experience derived from my own investigative and creative path during the doctoral pursuit, and in contrast with my experience in creative processes as a professional artist. Among the transformations that are in play today, also there is the willing to give back the meaning to the concept of knowledge, which would be interwoven to what we are. Knowledge would mean, first of all but not exclusively, a self-awareness, a self-transforming experience that in one way or another can make us "wiser" (more prudent, in the sense of the Aristotelian phronesis). In that sense, perhaps it could be said that to be a Doctor should not only mean to master the methods of the anonymous enterprise that is science; but also, and especially, it means to accomplish a self-transformative experience. To become a Doctor should also mean to reach a certain kind of virtue, to have been transformed from a certain kind of person into a better one.
\end{abstract}

Keywords: research-creation, doctoral training, visual arts, design.

\footnotetext{
1 Licenciada en Artes Visuales, Doctora en Diseño y Creación de la Universidad de Caldas, Fundación Universitaria del Área Andina, Pereira, Colombia. E-mail: silviaart@hotmail.com
} 


\section{Introducción}

Es una tarea relativamente reciente a la que se han dado los/as investigadores en los campos creativos al construir bancos con los enfoques y las perspectivas metodológicas aplicadas en el arte y el diseño; relacionar la investigación en estos campos disciplinares con conceptos presentes en las áreas de las ciencias naturales y sociales; proponer metodologías para dominios específicos; desarrollar competencias teóricas y disciplinares para la elaboración de proyectos; crear sistemas de análisis del pensamiento visual y modos de trabajo inter, multi y transdisciplinar. Pero aun así, falta definir con más precisión las particularidades de lo que implica investigar basados, mediados, guiados por la práctica, o como se le denomina actualmente, práctica como investigación (Sullivan, 2005; Hernández, 2006; McNiff, 2013; Nelson, 2013); especificar las destrezas y áreas del conocimiento que debe poseer un investigador en el campo de las disciplinas creativas (Newbury, 2012), determinar cuáles son los criterios que validan la práctica como investigación y entender la forma que toma el conocimiento cuando deriva de la práctica (Borgdorff, 2005).

Frayling (1993) publicó un artículo bajo el nombre de Investigación en arte y diseño, dejando entrever tres modos en los que se puede hacer investigación y que hoy todavía continúan siendo referidos: i) investigación para el arte y el diseño, es decir, que está al servicio de los objetivos de la práctica; ii) investigación en el arte y el diseño, esto significa que su objeto de estudio es la práctica; iii) investigación a través del arte y el diseño, donde la práctica del arte y del diseño sirven a un propósito de la investigación.

Basta con observar el texto: El debate sobre la investigación en artes, del profesor holandés Henk Borgdorff (2005) para identificar la cristalización de las reflexiones de Frayling en la "investigación en las artes", ruta propuesta por el autor para otorgarle un valor equitativo a la teoría y a la práctica mediante una relación de reciprocidad, que en el fondo viene siendo una preocupación por la relación entre investigación y creación.

La especialista en la práctica del arte Reilly (2002), ha hecho manifiesto que el modo de encarar una discusión sobre la práctica en el contexto universitario es, primero, aclarando qué se entiende por conocimiento en el arte y de qué manera la práctica puede adoptar forma en la investigación; segundo, dejando de lado la asociación del saber con la certeza, dado que el arte no tiene por objetivo conseguir certezas; y tercero, concediendo a la práctica, la imaginación y la sensibilidad un lugar dentro de los debates sobre la naturaleza del conocimiento. Pues el asunto medular es mucho más complejo que coexistir a la sombra del método científico, como ha sido hasta la actualidad, tiene que ver con la pregunta por ¿cómo se investiga y se genera conocimiento en la práctica?

Deforma constante,los/las artistas/investigadores/ docentes somos interpelados/as por la comunidad académica frente a cuestiones como: ¿Cuál es el lugar de la investigación en la creación y cuál el de la creación en la investigación académica? ¿De qué manera se investiga y se genera nuevo conocimiento en las disciplinas de la creación, de tal modo que pueda ser medido y evaluado por la investigación académica? ¿Las investigaciones que involucran la creación en el proceso de investigación son realmente investigaciones académicas? ¿Cuál es la diferencia entre investigación y creación? ¿Qué tipo de definición de investigación es la que reclaman las disciplinas de la creación en la actualidad? ¿Cuáles son los medios empleados por la investigación-creación para sustentar sus resultados y procesos? ¿Se ha logrado reconocer como válidas las experiencias de la creación en la definición de conocimiento? En este artículo se tratará de responder algunas de estas cuestiones. 


\section{Contexto investigativo}

Haber puesto en marcha una propuesta de investigación-creación en el marco de un doctorado supuso hacerse cargo de la producción de la obra artística, la invención de dispositivos de interacción humana, la reflexión y sistematización a través de la escritura académico-creativa, y la socialización de la experiencia y los acontecimientos creativos. Pero también significó haber entendido que una propuesta de creación que se adscribe a una investigación doctoral tiene por objetivo colmar de sentido la situación que estudia y no solo hallarlo.

Reclamar la pertinencia de la investigacióncreación en el campo de las artes en Colombia, es una discusión planteada por los/las creadores/ investigadores/docentes en el entorno universitario, porque es allí donde se objeta su valor y utilidad.

\section{Discusión}

La universidad tradicional ha sido, como lo defiende el pensamiento moderno, el recinto del conocimiento científico (Brea, 2007). Cualquier otro tipo de conocimiento que no se configure bajo las reglas teóricas de la cientificidad, es descalificado por la normatividad de los saberes hegemónicos.

Foucault (2000) reconoce el saber de la gente y los saberes que no fueron incluidos o que fueron enmascarados por los saberes hegemónicos, como saberes sometidos. En tal sentido, las artes frente al régimen del conocimiento científico, se presentan como saberes enmascarados dentro del terreno de las ciencias sociales y humanas, o sea de la ciencia, y sometidos a unas rutinas metodológicas, conceptuales, teóricas y discursivas que difieren radicalmente de la manera como se organiza, se produce y se comunica el conocimiento de la creación.
El debate sobre la creación en la universidad ha suscitado la generación de documentos que discuten la riqueza epistemológica de la práctica, pero también ha develado el funcionamiento anacrónico de la universidad en la contemporaneidad.

Los dispositivos de teorización del conocimiento sujetos a la forma del paper o artículo científico, la resistencia a hacer aparecer los sujetos en la escritura académica y en los fenómenos de estudio, el registro controlado y lineal del conocimiento y la didáctica de la economía discursiva, son algunos de los criterios que la universidad está llamada a revisar de cara a la forma que toma el conocimiento en la creación, y que Richard (2001) atiende en La cita académica y sus otros.

A pesar de que Richard (2001) plantea su aporte dentro del campo de los estudios culturales y la crítica cultural, el fondo de la discusión se adapta perfectamente al ámbito de la creación. Según la autora, "el diseño de habla y composición del saber" (p. 123) o lo que llamaríamos la puesta en forma del conocimiento científico, es uno de los elementos susceptibles de reinvención frente a los saberes locales o sometidos.

La indefinición o desaparición de las personas que elaboran el conocimiento es un rasgo característico en los papers científicos (Richard, 2001):

(...) la regla objetivadora del saber académico cuya pretensión de validez y sistematicidad se apoya generalmente en la in-definición de la persona [...] garantiza la abstracción del metadiscurso filosófico y científico encargado de trascender el precario detalle de la contingencia enunciativa del sujeto, borrando de la verbalidad el accidente de sus modos, tiempos, voces, números, personas y géneros (pp. 124125).

Borrar al sujeto y sus modos de ser sujeto del texto académico reclamando objetividad, es una discusión rebatida por Haraway (1995) en Los conoci- 
mientos situados. De acuerdo con esta teórica de la ciencia, el sujeto que da cuenta del conocimiento en un paper es en primer lugar un sujeto cargado de las marcas identitarias individuales y de las del universo simbólico y comunitario al que pertenece. Por lo tanto, es un sujeto compartimentado en múltiples dimensiones individuales y sociales y afectado por ellas. Y en segundo lugar, las observaciones a través de dispositivos de visualización y sus consecuentes análisis, son resultado de una interpretación subjetiva y de todo lo que incide en esa subjetividad (Haraway, 1995).

Los formatos de escritura que hacen visible al sujeto y las impresiones sensibles, temporales y espaciales de su cuerpo no solo son incompatibles con el paper, se reconocen por la jerarquía científica como formatos no prestos a la discusión académica, porque rompen con el protocolo científico de formalización y transmisión del conocimiento. Esto no debe confundirse con los productos de la creación artística.

Según la Secretaría de Cultura, Recreación y Deporte et al. (2013) no todos los productos materiales e inmateriales de la creación (obra) actúan como dispositivos de transmisión del saber en el sentido de la sistematización de conocimiento.

(...) no es la objetualidad de la obra lo que da cuenta como soporte único de la formalización de conocimiento, esta objetualidad -en caso de darse- puede reconocerse por medio de un sistema de documentación que sirva como forma paralela de existencia y de formalización del conocimiento, y al mismo tiempo permita construir la memoria de la experiencia y posibilite la construcción de archivo, fundamental para insertar el conocimiento en las redes de consulta (p. 57).

Pues bien, esto no se trata de un acto desobediente o subversivo. La autoridad académica del modelo científico en nombre de la objetividad, la claridad y la economía discursiva que rechaza lo que Richard (2001) llama las "errancias y desvíos poéticos" del texto y las extensas divagaciones, es también lo que ha obligado a las diferentes disciplinas artísticas y a los/las creadores/investigadores/docentes a formular sus propios protocolos de presentación del conocimiento o, al menos, unos formatos de escritura coherentes con la naturaleza del conocimiento que se produce en la creación.

El predominio del paper, según Richard (2001), sacrifica -desde la perspectiva del creador escritor-

(...) la espesura retórica y figurativa del lenguaje en el sentido (fuerte) de lo que Barthes llamaba "la teoría como escritura", es decir, la teoría que piensa sus formas y dice cómo se dice, para desinstrumentalizar el simple "referirse a" del saber práctico con palabras que retienen, en su urdimbre reflexiva, la memoria del deshacer y del rehacerse de la significación (p. 142).

Pero también sacrifica las imágenes en el sentido estético, visual, simbólico, político y cognitivo que ellas tienen para el arte. Documentar desde la imagen (fija, cinematográfica, videográfica y electrónica) es otra forma de construcción de la memoria $y$, por consiguiente, de conocimiento (Secretaría de Cultura, Recreación y Deporte et al., 2013).

Lo otro es que los productos de la creación no pueden considerarse estrictamente divulgadores del conocimiento (Secretaría de Cultura, Recreación y Deporte et al., 2013). Agrupar la producción artística en la categoría de divulgación, como lo propuso Colciencias en el sistema colombiano de investigación, es restarle valor a lo que la creación configura por sí misma, independientemente de si se acoge o no a la maquinaria universitaria. Esto entraría en total desajuste con la creación producida por fuera del ámbito universitario que no aspira al reconocimiento de la comunidad académica. De allí que una de las cuestiones a resolver en este artículo sea la diferencia entre una propuesta de creación que se hace en el marco de un doctorado, es decir, en el entorno académico, y la propues- 
ta de creación que se hace como resultado de la práctica cotidiana del artista.

La investigación-creación y su relación con la universidad es una puesta en forma del conocimiento que produce la práctica articulada a ese ensamblaje académico. Con ello no digo que la función o la naturaleza de la creación en la universidad tenga que derivar en un engranaje horizontal dentro de la dupla investigación-creación. De hecho, el esfuerzo de algunas universidades colombianas y de sus sistemas de investigación consiste en darle prioridad a la creación reconociendo en la práctica su valor cognitivo. Pero tampoco estoy diciendo que la creación que no se articula a esa formalización de la dupla no produzca conocimiento.

Borgdorff (2005) ha definido cuándo la práctica artística se califica como investigación a partir de tres aspectos: la ontología, la epistemología y la metodología.

La práctica artística puede ser calificada como investigación si su propósito es aumentar nuestro conocimiento y comprensión, llevando a cabo una investigación original en y a través de objetos artísticos y procesos creativos. La investigación de arte comienza haciendo preguntas que son pertinentes en el contexto investigador y en el mundo del arte. Los investigadores emplean métodos experimentales y hermenéuticos que muestran y articulan el conocimiento tácito que está ubicado y encarnado en trabajos artísticos y procesos artísticos específicos. Los procesos y resultados de la investigación están documentados y difundidos de manera apropiada dentro de la comunidad investigadora y entre un público más amplio (p. 27).

Lo ontológico toma cuerpo en tanto da por hecho que el núcleo de la investigación es el proceso creativo y todo lo que él abarca en cuanto a resultado (material e inmaterial) y contexto (entorno de recepción e interpretación o mundo del arte); lo epistemológico, en tanto la práctica genera un conocimiento cuya naturaleza reside en los artefactos y los procesos derivados de esa práctica; y lo metodológico, en tanto hace visible la particularidad y necesidad de unos métodos y técnicas que incorporen la experimentación e interpretación de la práctica para darle sentido a un conocimiento que podrá ser tácito, práctico, sensorial, no conceptual o no discursivo (Borgdorff, 2005).

Esta estructura así descrita por Borgdorff (2005) avala la pertinencia de que en cualquier unidad académica, doctorado como en este caso, una propuesta de creación artística pueda valorarse -en el sentido riguroso- como una propuesta de investigación académica, cuyo objeto es ampliar el conocimiento. También introduce una reflexión importante sobre el hacer cuando enfatiza en que "crear [...] forma parte del proceso de la investigación" (Borgdorff, 2005, p. 24). Esto significa que la práctica comprendida como el eje central del pensamiento teórico, del ejercicio de indagación y de la materialización sensible de la creación posibilita procesos de búsqueda, sistematización, documentación, materialización y comunicabilidad del conocimiento.

Por supuesto, círculos académicos más escépticos desaprueban la práctica argumentando que "el impacto de la investigación queda confinado a la propia obra del artista" (Borgdorff, 2005, p. 25). Pero esto solo tiene lugar si dicha práctica no se adscribe a un contexto más amplio de la investigación, o si su objetivo no es la investigación. En tal caso, su repercusión no alcanzaría un ámbito más extenso que la del propio arte como es el caso de la práctica cotidiana del artista que no se adhiere al terreno académico.

Ahora bien, ¿de qué forma la investigación-creación se ocupa de organizar la comprensión de una actividad que por definición es metafórica, alegórica, simbólica, subjetiva y estética? Que la práctica se legitime como investigación y responda a sus objetivos, no la exime de su carácter estético y mucho menos, del interés y la incidencia que 
esta podrá tener en el ámbito del arte y sus alrededores. Así como tampoco el artista que no se adscriba a una estructura académica queda relegado de la actividad investigativa. Y al hilo de esta discusión, ¿qué tipo de obras son las que produce la investigación-creación? y ¿qué las diferencia de otros tipos de producción estético-artística?

La investigación-creación es un modelo de relación entre los seres humanos y sus contornos de saber, que forma y deforma el conocimiento sensible desde lo individual, lo grupal y lo social. Lo que sus artefactos y prácticas producen son procesos de comprensión sobre el funcionamiento de las sociedades. En ese sentido, la organización e instalación de esas obras o prácticas estético-artísticas dependerá primero de la manera particular como cada artista configura su hacer y da cuenta de su proceso y, segundo, de los lugares o ambientes en los que esa producción produce sentido: el museo, la calle, la fiesta, la web, la universidad, el simposio, el laboratorio, etc.

Lo que hoy reclaman las disciplinas de la creación no es una nueva definición de investigación que obligue a la ciencia a actualizarse, aunque debería, es una definición de investigación que les permita existir en la singularidad de su condición cognitiva de lo sensible, donde confluyen la imaginación, la intuición, la experiencia íntima, la sensibilidad, lo intersubjetivo, lo cotidiano, entre otros, como valores propios a la naturaleza de ese conocimiento.

En materia de investigación en artes, la academia, los gestores, las instituciones y los/las artistas/investigadores/docentes nos hemos dado a la tarea de configurar una definición sobre investigación en artes, que nos ayude a obtener el merecido reconocimiento de una labor cuyo objeto es producir sentido a través de la aplicación de un conocimiento sensible. Definición que ha motivado la construcción de un aparato conceptual que integre la práctica al sistema de investigación y, que a su vez, ponga en tensión la naturaleza de la investigación practicada en la universidad.
Investigación-creación ha sido el término adoptado por algunos/as artistas/investigadores/ docentes para referirse a la investigación en artes en Colombia. Algunos autores/as han hecho manifiesto que la investigación-creación es el método a través del cual el arte genera conocimiento $y$, así mismo, un caballito de batalla para insertarse en la sociedad del conocimiento (Daza-Cuartas, 2006). Otros, por el contrario, consideran que es un viaje a ninguna parte, en el que los propios artistas se han dedicado a "imponerle a la producción artística la condición de estar ligada a un proceso de investigacióncreación, a fin de validarla institucionalmente, desnaturalizando la creación artística" (OsunaBarriga, 2012, p. 8).

Razón por la cual teóricos/as de la práctica de las artes se han visto en la obligación de argumentar la importancia de la palabra "investigación" en la dupla investigación-creación que obedece, por un lado, al alcance que puede tener el conocimiento dentro de una comunidad heterogénea si se comunica en clave investigativa, y por el otro, a una forma de validar y de insertar la investigación artística en las agendas nacionales e internacionales de la investigación, que entre otras cosas, permite el reconocimiento de protocolos adecuados y metodologías situadas en contextos y cuestiones de interés para las disciplinas de la creación (Asprilla, 2013).

Hay quienes invierten la palabra investigación en la dupla reafirmando la creación como categoría mayor, con el fin de devolverle el carácter cognoscitivo a la creación y de preservar la creación como forma privilegiada de producción en las artes, en la cual la investigación es solo una manera de poner a circular el conocimiento (Asprilla, 2013).

Tenemos, de un lado, autores que han caracterizado los tipos de artistas que coexisten en esa coalición (Martínez-Vesga, 2006): 1. Los que hacen documentos, sistematizan y no tienen ningún interés en encajar en la academia o en 
lugares ajenos a la práctica artística; 2. Los que producen obra y sistematizan sus procesos de reflexión y producción técnica; y 3 . Los que se comprometen con su campo de producción y materialización artística, pero no sistematizan sus procesos.

De manera paralela, autores que desde otras latitudes geopolíticas han tratado de configurar rutas de trabajo cuando la práctica y el hacedor se permean por la investigación (Fajardo-González, 2008): 1. Una investigación de la cual al final sale una creación como apéndice; 2 . Una investigación que transcurre paralelamente con la creación; 3. La creación como protagonista donde se le adapta el discurso de la investigación.

$Y$ de otro lado, se encuentran los/las autores que nos muestran los Sistemas de Investigación de Facultad (SIF), de incentivos y de cualificación de las universidades que interiorizaron la investigacióncreación como modelo y lo pusieron en marcha (Santamaría-Delgado et al., 2011; Moreno-Vargas, 2012; Vélez-Cuartas, Gómez-Flórez, Úsuga-Ciro, \& Vélez-Trujillo, 2014).

En el artículo: La investigación-creación en el contexto de las prácticas estético-artísticas contemporáneas: desplazamientos disciplinares y desafíos institucionales, Calle (2013) reconoce tres grandes paradigmas de producción que se imponen en el campo de las prácticas artísticas contemporáneas: 1. La obra única, 2. La multiplicación y fragmentación del objeto artístico y 3 . El archivo como sistema de memoria material. En este esfuerzo teórico Calle (2013) da cuenta de los tránsitos y desembocaduras de las prácticas artísticas en el terreno de la investigación y del propio arte. Sin embargo, es necesario hacer visibles las tensiones inherentes a este modo de producir conocimiento que conciernen al rigor: una cosa es el rigor de la investigación académica y cientificista y otra el rigor de la creación.

a. Toda investigación inicia con la identificación de un problema. Mientras que la génesis de un proce- so de creación tiene múltiples posibles inicios que no son precisamente a partir de un problema. $Y$ al respecto argumentan Gil y Laignelet (2014) en el marco del II Encuentro sobre Creación, Pedagogía y Políticas del Conocimiento:

Puede iniciar desde una afirmación emocional, [...] desde una impresión sensorial, desde un elemento perturbador en la memoria o en la psiquis, puede empezar desde un instante altamente significativo, puede empezar también desde lo inconsciente, desde un dato onírico. No necesita justificación. Y también, por supuesto, puede empezar desde una pregunta.

Con el protocolo de creación-investigación que la pianista Ligia Asprilla (2013) aporta en Los productos de la creación-investigación: la producción de conocimiento desde las artes, logra mediar entre las etapas que no pueden estar ausentes en la investigación académica y las que no pueden estar ausentes en la creación. Aunque no introduce una etapa con el nombre de planteamiento del problema, si propone lo que ha llamado el objeto de creación, que no son más que los motivos e ideas en los que se centrará la indagación, generándose un documento prolífico en el que resulta válido optar por el desarrollo de una investigación, cuyo objeto de estudio son los procesos de la creación, los elementos de la creación, los contextos de la creación, los campos articulados al arte, los lenguajes artísticos, el proceso de creación de una obra, las prácticas culturales y/o las metodologías, didácticas y pedagogías del arte (Asprilla, 2013).

De lo que estamos hablando es de investigaciones sobre la creación, a través de la creación, en la creación, para la creación. Cuestión que ya había introducido Frayling (1993) y que es ampliada por Borgdorff (2005) en La investigación en arte, por Hernández (2006) en la Investigación Basada en las Artes y por Nelson (2013) en La práctica como investigación en las artes, solo por mencionar algunos teóricos. 
b. La investigación tiene por regla la elaboración de respuestas afirmativas y/o negativas (hipótesis) que son sometidas a una metodología de comprobación. El arte en cambio no elabora hipótesis y tampoco sigue metodologías de comprobación. Su conocimiento se mueve "hacia el ámbito de las experiencias singulares", según Gil y Laignelet (2014). Por lo tanto, su única certidumbre es la incertidumbre.

De lo que sí se hace cargo el arte es de la especulación, incubación y formulación de preguntas de investigación que se relacionan con cuestiones propias de su conocimiento o del ámbito que estudia, en las que se expresan inquietudes y curiosidades de corte estético, creativo, pero también epistemológico.

c. La metodología que opera en la investigación sigue un conducto lineal y ordenado que posibilita la corroboración de las hipótesis. En la creación, las tácticas, exploraciones y estrategias serán equiparables a la metodología. En todo caso, procesos que no atienden a un ordenamiento lineal. Lo cual no quiere decir que no existan unas etapas conducentes a otras.

En lo que concierne a la investigación-creación, Osuna-Barriga (2012), acudiendo a los enfoques cognitivos, describe dos fases que configuran el acto creativo: fase 1 o fase generativa y fase 20 fase exploratoria. En la fase generativa se originan procesos de memoria, transferencia analógica, aparición de formas de objetos, modelos mentales y patrones que en la fase exploratoria quedan sometidos a interpretaciones conceptuales, búsquedas de atributos, categorías y límites. El objeto o producto de creación podrá aparecer en la fase 1 o solo después de cruzar la fase 2 o, incluso, podrá expandirse cuanto lo necesite. De allí la incertidumbre.

Osuna-Barriga (2012) enfatiza en dos cuestiones relevantes: la primera, en la importancia de no ingresar a la fase generativa con un amplio grupo de categorías, funciones o tipologías que cierren o limiten el espectro inventivo y por lo tanto, prefiguren el objeto final; y la segunda, en la nutrida antesala que la especulación hace a la fase exploratoria donde, muchas veces, cobra forma el producto final.

La metodología adecuada, asegura Fajardo-González, (2008) es aquella que aborda y reflexiona los propios procesos creativos que dan origen a la obra de arte y a todo aquello en lo que podría incidir: el universo de la experiencia humana, la sociedad y la existencia cultural.

d. La objetividad y la racionalidad son propósitos que persigue la investigación para poder controlar los objetivos y los resultados. Que la creación camine a través de la incertidumbre parece un acto descontrolado y desorganizado. Pero que su conocimiento dé cuenta del sujeto y de su cuerpo en relación con el mundo como el contexto de estudio, y que a su vez, ese mismo sujeto sea quien ponga la mirada sobre sí mismo y sobre los demás, es completamente irracional y subjetivo.

Frente a esta dualidad, la investigación-creación aporta una variación no solo del mundo que conocemos sino del modo como lo conocemos, que exhorta a la comprensión de una subjetividad y de su condición multidimensional, donde cada compartimento está conectado a la totalidad de todas las experiencias y facetas de la condición humana, de lo imaginario y lo racional, de lo abstracto y lo concreto.

Con lo anterior, la investigación-creación esboza su embrionario sentido de subjetividad, entendido por Haraway (1995) como la importancia de aprender en nuestros cuerpos. Si el conocimiento es un entramado de cuerpos y lenguajes, el ojo humano es el vehículo entre la experiencia del cuerpo en relación con el mundo, y será sobre la subjetividad y la experiencia humana de lo que dé cuenta el ojo. Lo que precisa ese avistamiento es el reconocimiento de la multiplicidad de los conocimientos tanto como el de las identidades que los producen (Haraway, 1995). 
e. La sistematización y divulgación del conocimiento en la creación no es de carácter explicativo, mas bien despliega en el lenguaje niveles polisémicos y polivalentes; pero también migra hacia otras formas de sensibilidad crítica distinta al lenguaje. ¿O acaso las libretas de artista de Leonardo o los diarios de ruta que usaron Julio Cortázar y Carol Dunlop para su proyecto Los autonautas de la cosmopista, no reflejan un ejercicio juicioso de sistematización de los procesos de observación y contacto con la realidad? (Osuna-Barriga, 2012). Por la pluralidad de sus prácticas, la riqueza discursiva del arte se expande hacia plataformas retóricas, expresivas, metafóricas, narrativas y artefactuales que le permiten existir en lugares territoriales y extraterritoriales.

Sin embargo, la discusión sobre la transferencia de conocimiento no solo se asienta en la manera como se sistematiza en la investigación o en la creación, o de los lugares de arraigo, que también son muy relevantes, pues de los lugares donde se instale ese conocimiento dependerá el usufructo que pueda obtenerse de él.

Se irradia hacia la distinción entre la experiencia estética y la transferencia de unos resultados de carácter académico, es decir, en la distinción entre lo que propone un artista cuya práctica confluye en ámbitos ajenos a la investigación académica, y lo que debe proponer un artista como investigador cuando su práctica artística se adhiere a los propósitos de la investigación. Cabe preguntarse si cualquier práctica artística es investigación.

f. Los productos resultantes de la investigación adoptan la lógica discursiva, buscan el consenso ante una comunidad científica y son medibles mediante criterios cuantitativos. En la creación los productos se valoran por "el disenso, la heterogeneidad de interpretaciones, por las diversas formas en que el sujeto determina el sentido" (Gil \& Laignelet, 2014). La dicotomía entre objeto y sujeto se acorta o no existe en la creación, lo cual permite al sujeto definir qué sentido tiene la experiencia y en qué modo resulta transformadora para él y sus congéneres.

Sin embargo, los debates por la medición como mecanismo de inclusión o exclusión, no se centran tanto en los criterios como sí en la tipología de productos que se dejan por fuera de la construcción del conocimiento, y que necesitan ser valorados por unos pares académicos con las competencias para interpretar, problematizar y criticar la creación. Esto lo demuestra un estudio reciente adelantado por Vélez-Cuartas et al. (2014), bajo el nombre de Diversidad y reconocimiento de la producción académica en los sistemas de evaluación en Colombia, llevado a cabo en las seis universidades nacionales (Universidad Nacional de Colombia, Universidad de Antioquia, Universidad del Valle, Universidad de los Andes, Universidad del Rosario y Pontificia Universidad Javeriana) mejor posicionadas en los rankings internacionales, que concluye con la existencia de al menos 70 formas diferentes de producción del conocimiento en las artes no reconocidas por Colciencias y no visibilizadas en el debate actual.

Un problema destacado, no solo dela investigacióncreación sino del arte contemporáneo en general, es el hecho de que al parecer se liquida toda posibilidad de juzgar o valorar. Jiménez (2010) se pregunta en La querella del arte contemporáneo:

¿Existen aún criterios de apreciación estética? [...] ¿Es posible redefinir las condiciones de ejercicio del juicio estético frente a las obras contemporáneas? Suponiendo incluso que estas últimas fuesen 'cualquier cosa', ¿se puede sostener un discurso argumentado y crítico sobre ellas? [...] ¿Cómo juzgar la calidad artística de objetos y prácticas si ya no existen criterios ni normas a los cuales remitirse? [...] la paradoja de la situación creada por el arte contemporáneo radica no solo en la indefinición del arte, sino también en el hecho de que la palabra 'arte' implica, pese a todo, no obstante su indeterminación, un juicio de valor (pp.13-34). 
Y no sugiero que calificar un producto de investigación-creación sea necesariamente equivalente a lo que hace la crítica de arte, pero de todos modos, ¿dónde trazar la diferencia? ¿Qué aspectos debe considerar un evaluador de un producto de investigación-creación y en qué difieren de los que debe considerar un crítico?

g. La preocupación, ahora que se mencionan los pares académicos, también se extiende al entrenamiento y a los compromisos investigativos que debe recibir y proyectar, en este caso un doctor en arte y diseño, quien será el más cualificado, así como el replicador de casos exitosos desde la experiencia investigativa y creativa (o al menos eso se espera), para formular juicios investigativos sobre esa producción que se genera en el ámbito de la creación.

Si el entrenamiento que recibe un doctor en cualquier otra disciplina es el de la investigación, lo primero que hay que tener claro es qué significa investigar. De acuerdo con el profesor británico Newbury (2012) la investigación requiere de dos componentes vitales: ideas y evidencias, pero no son cualquier tipo de ideas ni cualquier tipo de evidencias, porque ideas sin evidencias son apenas especulaciones y evidencias sin ideas es información sin sentido. Además de una relación dialógica entre las ideas y las evidencias y una configuración de las mismas desde los recursos de las artes y el diseño.

A esto se suman tres compromisos significativos de toda investigación doctoral: la transparencia metodológica, la comunicabilidad del conocimiento y el imperativo pedagógico. Las dos primeras tiene que ver, según Newbury, con dar cuenta por escrito y de forma oral a través de ejercicios conjuntos e individuales que articulen suficiencia teórica y crítica -en coloquios, encuentros, foros y sustentaciones a comités científicos- sobre el avance de la investigación en particular, y de la manera como se construye el conocimiento en general. En otras palabras, la transparencia metodológica y comunicabilidad significan que el conocimiento impli- cado en la investigación no es, digamos así, de tipo esotérico, como en el misticismo, no se trata de verdades reveladas a las que se accede solo por vía de una transformación interior.

Mas bien pareciera que de nuevo esto nos conecta con la valoración de los productos de la investigación-creación, porque el asunto aquí es más o menos este: ¿hasta qué punto es en realidad posible que se genere un debate "racional" respecto de un producto de investigación-creación? ¿Será que la única actitud adecuada a este tipo de productos es la "contemplación" o la experiencia estética? Pero, de otro lado, dicha transparencia ¿implica que el artista debe estar en condiciones de explicar cómo fue posible el proceso de creación, o debe incluso ser "crítico" de su propia obra, debe hacer algo así como el absurdo de tratar de "explicarla"?

Y con el imperativo pedagógico se refiere a aprender a hacer investigación. Pero aprender a investigar no se reduce a un proceso de revisión y recolección de autores, teorías y datos, ni tampoco a la fabricación arbitraria de evidencias, sino a una reflexión permanente y crítica por la manera como se aborda el proceso mismo de la investigación (Newbury, 2012).

Reconocer la existencia de este ensamble entre la creación y la investigación -donde articular la teoría y la práctica dependen, por un lado, del proceso creativo (entiéndase obra) y, por el otro, de la reflexión crítica de los fenómenos que intervienen y participan de ese proceso creativo, pero también de todos los casos, metodologías y protocolos usados por los/as artistas investigadores- es la primera tarea de la que tendrían que ocuparse los programas de doctorado en el país. Además de comprender las particularidades de la investigación-creación y de definir los alcances académicos y creativos de una forma de producción de conocimiento académico, que pone la práctica en el centro de la discusión teórica y la formalización estética. 


\section{Conclusiones}

Lo realmente valioso de este tipo de investigación, que reclama una actitud crítica y democrática de quienes se reconocen en el ejercicio de la investigación, es la configuración de nuevas entradas o maneras de llegar al conocimiento. Maneras que vean en el arte, en lo sensible, lo intuitivo, lo pluriforme, lo incierto, lo imaginativo, la posibilidad no solo de transformar la realidad sino el camino para comprender y valorar lo humano.

Cabe decir, como lo demuestra el debate, que las disciplinas de la creación no se han marginado de la esfera académica de la investigación porque carezcan de relaciones conceptuales, teóricas o epistemológicas, sino porque la estrechez de la definición de investigación proveniente del saber científico ha dejado por fuera la contribución de la práctica.

Si bien la investigación-creación es la dirección en la que se están formulando los procesos de producción del conocimiento en algunos programas de pregrado y posgrado en arte, diseño y arquitectura, la ruta aún no está legitimada por Colciencias. Reclamar la existencia del conocimiento sensible y de la investigación-creación como el modo en que ese saber toma forma en el ámbito universitario, nos enfrenta a preguntarnos qué es lo que hay que demostrar, ¿que el arte produce conocimiento o que el conocimiento es más universal y debe ir más allá de la forma que cada campo le da al saber?

Tal vez de lo que se trata es de entender que la ciencia, el arte y la técnica no son elementos aislados o sin referencia al conjunto de una cultura o del saber, y que las relaciones entre ellas -como esferas autónomas y transversales del conocimiento- son cambiantes y móviles. Generar fisuras, desacomodos y reordenamientos conceptuales, simbólicos y creativos es parte del ejercicio de imaginarnos y de actuar como sujetos sociales de conocimiento en el marco de la universidad.
Me parece que, sin la pretensión de que esto lo agote, estamos obligados a reconstruir una visión integradora del saber, y esto implica que el artista debe comprenderse a sí mismo como "científico", como un intelectual, como alguien que no solo se dedica a la creación, en el sentido mas bien formal del término, sino como alguien inquieto, curioso, que toma posición frente al mundo y la vida, que tiene algo qué decir.

\section{Agradecimientos}

Este artículo de reflexión derivada de la investigación doctoral: "Creación e Investigación: La práctica artística contemporánea en el contexto de las identidades transgénero como un proceso transformador, trans (vesti) y transdisciplinar"; llevada a cabo en el Doctorado en Diseño y Creación de la Universidad de Caldas y financiada por Colciencias, a través de la BECA-CRÉDITO Convocatoria 567 Doctorado Nacional.

\section{Referencias}

Asprilla, L. (2013). Los productos de la creacióninvestigación: la producción de conocimientos desde el arte. Bogotá, Colombia: Asamblea General de la Asociación Colombiana de Facultades y Programas de Artes- ACOFARTES.

Borgdorff,H. (2005). El debate sobrela investigación en artes. Encuentro Arte como Investigación en Félix Meritis. Encuentro llevado a cabo en Amsterdam, Holanda. Recuperado de http://www.ahk.nl/ lectoraten/onderzoek/ahkL.htm

Brea, J. L. (2007). Cultura RAM. Mutaciones de la cultura en la era de su distribución electrónica. Barcelona, España: Gedisa.

Calle, M. (2013). La investigación-creación en el contexto de las prácticas estético-artísticas contemporáneas. Desplazamientos disciplinares y desafíos institucionales. Mediaciones Sociales, 12, 65-79. doi: /10.5209/rev_MESO.2013.n12.45263 
Daza-Cuartas, S.L. (2009). Investigación-creación. Un acercamiento a la investigación en artes. Plumilla Educativa, 11 (1), 87-92. Recuperado de: http://revistas.iberoamericana.edu.co/index.php/ rhpedagogicos/article/view/339

Fajardo-González, R. (2008). La investigación en el campo de las artes visuales y el ámbito académico universitario. Societas, 10 (2), 85-98.

Foucault, M. (2000). Defender la sociedad. Curso en el Collège de France. Buenos Aires, Argentina: Fondo de Cultura Económica de Argentina. (Trabajo original publicado en 1975-1976).

Frayling, C. (1993). Research in art and design. Royal College of Art Research Papers, 1(1), 1-5.

Gil, J., \& Laignelet, V. (2014). Las artes y las políticas del conocimiento. Tensiones y distensiones. En II Encuentro sobre Creación, Pedagogía y Políticas del Conocimiento. Encuentro llevado a cabo en Bogotá, Colombia.

Haraway, D. (1995). Ciencia, cyborgs y mujeres. La reinvención de la naturaleza. (Manuel Talens, Trad.). Madrid: Cátedra. (Trabajo original publicado en 1991).

Hernández, F. (2006). Campos, temas y metodologías para la investigación relacionada con las artes. En Gómez, M., Hernández, F., \& Pérez, $\mathrm{H}$. Bases para un debate sobre investigación artística. Madrid, España: Ministerio de Educación y Ciencia.

Jiménez, M. (2010). La querella del arte contemporáneo. Buenos Aires, Argentina: Amorrortu.

Martínez-Vesga, O. (2006). Investigar, crear, experimentar el mundo. Reflexiones sobre la investigación en las artes plásticas. El artista, (3), 57-68. Recuperado de: http://www.redalyc.org/ articulo.oa?id=87400305
McNiff, S. (Ed.). (2013). Art as research. Opportunities and callenges. Chicago, Estados Unidos: Intellect Bristol.

Moreno-Vargas, C. A. (2012). El arte de la investigación-creación. Pesquisa, 15. Recuperado de http://www.javeriana.edu.co/pesquisa/wpcontent/uploads/pesquisa20_06.pdf

Nelson, R. (2013). Practice as research in the arts. Principles, protocols, pedagogies, resitances. Nueva York, Estados Unidos: Palgrave Macmillan.

Newbury, D. (2012). Research training in the creative arts and design. En Biggs, M., \& Karlsson, $\mathrm{H}$. The routledge companion to research in the arts. Nueva York, Estados Unidos: Routledge.

Osuna-Barriga, J.G. (2012). Un viaje a ninguna parte: la investigación-creación como un vehículo de validación institucional de la producción artística. Cuadernos de música, artes visuales y artes escénicas, 7 (1), 5-9. Recuperado de: http://www. redalyc.org/articulo.oa?id=297023537001

Reilly, L. (2002). An alternative model of "knowledge" for the arts. Working Papers in Art and Design 2. Recuperado de https://www. herts.ac.uk/_data/assets/pdf_file/0015/12309/ WPIAAD_vol2_reilly.pdf

Richard, N. (2001). Residuos y metáforas. (Ensayos de crítica cultural sobre el Chile de la Transición). Santiago de Chile, Chile: Cuarto Propio.

Santamaría-Delgado, C., Chingaté-Hernández, N., González-Betancur, J.D., Castellano-Camacho, N., Salazar-Ospina, M., \& Morales-Serrato, S. (2011). La productividad de las artes en las universidades colombianas: desafíos a los mecanismos de medición del conocimiento. Cuadernos de música, artes visuales y artes escénica, 6 (2), 87-116. Recuperado de: http://cuadernosmusicayartes. javeriana.edu.co/index.php?option $=\mathrm{com}_{-}$ content\&view=article\&id=90:la-productividad- 
de-las-artes-en-las-universidades-colombianasdesafios-a-los-mecanismos-de-medicion-delconocimiento\&catid=23:volumen-6-numero2\&ltemid $=10$

Secretaría de Cultura, Recreación y Deporte, \& Fundación Universidad de Bogotá Jorge Tadeo Lozano. (2013). El arte como productor de conocimiento (Convenio de Asociación № 209). Recuperado de: http://acofartes.org.co/docsweb/ investigacion/abril/Dic\%2013-13\%20Arte\%20 prod\%20de\%20conoc\%20IDARTES-TADEO.pdf
Sullivan, G. (2005). Art practice as research. California, Estados Unidos: Sage.

Vélez-Cuartas, G., Gómez-Flórez, H., ÚsugaCiro, A., \& Vélez-Trujillo, M. (2004). Diversidad y reconocimiento de la producción académica en los sistemas de la investigación en Colombia. Revista española de documentación científica, 37 (3), 1-14. doi:10.3989/redc.2014.3.1133 